\title{
Multi-resolution Image Analysis for Vehicle Detection
}

\author{
Cristina Hilario, Juan Manuel Collado, \\ José Maria Armingol, and Arturo de la Escalera \\ Grupo de Sistemas Inteligentes, Universidad Carlos III de Madrid \\ C/ Butarque 15, 28911 Leganés, Spain \\ \{chilario, jcollado, armingol, escalera\}@ing.uc3m.es
}

\begin{abstract}
Computer Vision can provide a great deal of assistance to Intelligent Vehicles. In this paper an Advanced Driver Assistance Systems for Vehicle Detection is presented. A ge ometric model of the vehicle is defin ed where its energy function in cludes infor mation of the shape and symmetry of the vehicle and the shadow it produces. A genetic algorithm finds the optimum parameter values. As the algorithm receiv es info rmation from a road detection modul e some geometric restrictions can be applied. A multi-resolution approach is used to speed up th e algorithm and w ork in re altime. Examples of re al images are shown to validate the algorithm.
\end{abstract}

\section{Advanced Driver Assistance Systems}

\subsection{Motivation}

Several Advanced Driver As sistance Systems (ADAS), t hat nowadays are bei ng researched for Intelligent Vehicles, are based on Computer Vision [1]. One of them has the goal of detecting and tracking other vehicles. Present day, commercial equipments are based on distance sensors like radar or laser. These sensors have the advantage of giving a direct distance measurement and, above all, they are able to work unde $\mathrm{r}$ bad weather conditions. Their main inconvenience is the field of view, which is very narrow, so they can only detect the vehicle in front of the sensor. If the vehicle is overtaken, there is a step input to the system and the response can be unstable. One alternative or complementary sen sor is vision. Although it is $\mathrm{n}$ ot able to work under bad weather conditions and its information is much difficult to process, it $\mathrm{g}$ ives a rich er description of the environment that surrounds the vehicle. Besides, many of the current traffic accidents happen under good weather and are due to human errors.

\subsection{Previous Work}

The research on vehicle detection based on an onboard computer vision system can be classified in three groups:

- Bottom-up. T here are some f eatures that define a ve hicle (symmetry, edges, shadow), and they are looked for sequentially in the image. Their main inconveniences are: the vehicle is lost if one feature is not present enough in the image and false tracks can deceive the algorithm. 
- Top-down. There are o ne or seve ral m odels of ve hicles and the best model is found in the image through a likelihood function. They are more robust than the previous al gorithms, but slower. The algo rithm presented in th is article fo llows this approach.

- Learning based. Mainly, they are based on ne ural networks. Many i mages a re needed to train the network. They are usually used in conjunction with a bottomup al gorithm to check if a vehicle has been actually detected. Otherwise, they have to scan the whole image and they are very slow

The shadow under the vehicles is looked for in [2]. To do so, a sample of the road just in front of the vehicle is taken and darker zones are searched. For these regions, symmetry and vertical edges confirm if there is a vehicle. A similar approach is found in [3]. In [4] a formula for symmetry is proposed. An elastic net is place $d$ at the maxi mum and it is deformed until the vehicle is found. Interesting zones in the image are localized in [5] using Local Orientation Coding. A Back-propagation neural network confirms or rejects the presence of a vehicle. [6] follows the previous work but adding texture and shadows. The tracking is done using the Hausdorff distance to a model . Another ex ample of fusing shadow, en tropy and symmetry is fo und in [7]. In [8], shadows and symmetry are proposed to localize in teresting zones; a neural network confirms the hypothesis. Symmetry is used in [9] to determine the column of the image where the vehicle is. After that, they look for an U-form pattern to find the vehicle. The tracking is performed with SSD correlation. They use a multi-reso lution approach. Edges and symmet ry are also use d in [10]. In [11] overtaking vehicles are detected through motion (i mage difference) and the other ve hicles through correlation. The dimension of the correlation window is calculated through edge detection. Several 3D models of vehicles are used in [12]. The road limits are calculated and the geometrical relationship between the camera and the road is known. Preceding vehicles are detected in [13 ]. They calcu late a Mu lticlustered Modified Quadratic D iscriminant Function through examples, and look for vehicles in regions of $16 \times 16$ pixels in the image.

\section{Geometrical Models}

As stated in [14], a global shaped model based image segmentation scheme consists of the following blocks:

- The initial model, $M$.

- The de formable model $M(Z)$. Thi s m odel is obt ained f rom the pre vious one through the deformation parameters, $Z$.

- The likelihood probability density function, $P(I \mid Z)$, which mean s the probability of the deformation set $Z$ occurs, in the image $I$.

- A search algorithm to find the maximum of the posterior probability $P(Z \mid I)$.

- The lik elihood function $P(I \mid Z)$ has t o be d esigned to rea ch i ts maxi mum val ue when the deformed model matches image $I$.

\subsection{Geometrical Model of a Vehicle}

Due to shadows, occlusi ons, weather conditions, etc, the model has to incorporate as much information as possible. In this paper, a vehicle is defined by seven parameters 


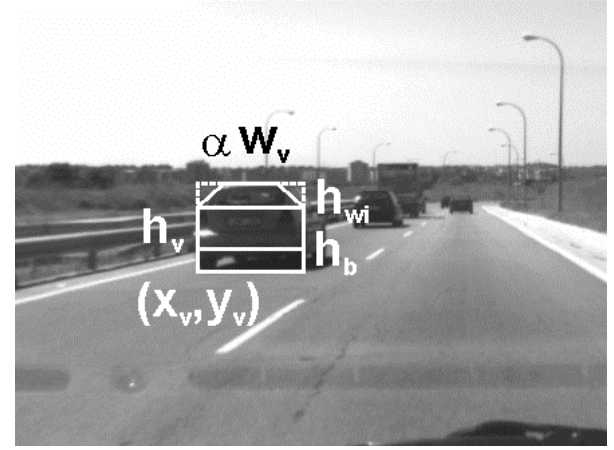

(a)

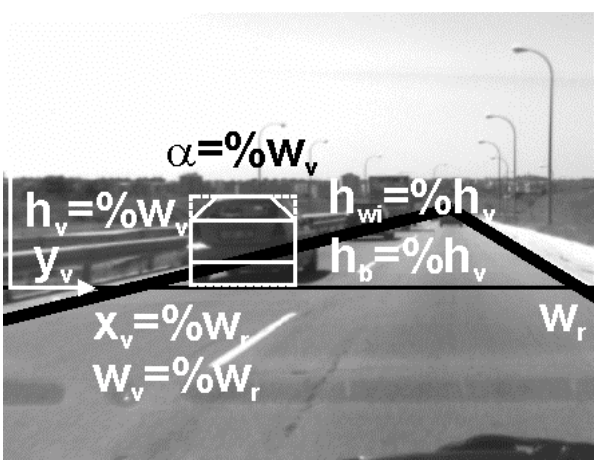

(b)

Fig. 1. Geometrical model of a v ehicle. (a) A v ehicle is defined by seven parameters: Position $(\mathrm{x}, \mathrm{y})$, width and height of the vehicle, windshield position, bumper position and roof angle (b) The values of this parameters are constrained by the detection of the road.

(Fig. 1-a): Position (x,y), width and height of the vehicle, windshield position, bumper position and roof angle. In a previous research, [15], the seven parameters had a range but, while the range of the $\mathrm{X}$ and $\mathrm{Y}$ position, and the width and height of the vehicle were in pixels, the range of the windshield and bumper position and the ro of angle were a percentage of the height or width.

A previous detection of the road limits is done in [2] [10]. This can help the vehicle detection step because the se arched area is smaller. In the present case, both borders of the road are found and modelled by equations:

$$
x=f_{l}(y) \quad x=f_{r}(y)
$$

that are the slope of the straight lines in this case, but the algorithm would be the same if they were parabolas or clotoids. For a specific $y_{v}$ value (Fig. 1-b), the width of the road is found:

$$
x_{l}=f_{l}\left(y_{v}\right) \quad x_{r}=f_{r}\left(y_{v}\right) \Rightarrow w_{r}=x_{r}-x_{l}
$$

The $x_{v}$ value of the vehicle and its width are two percentages of the width of the road:

$$
x_{v}=K_{x} w_{r} \quad w_{v}=K_{w} w_{r}
$$

The height of the vehicle is proportional to the width:

$$
h_{v}=K_{h} w_{v}
$$

And finally, the windshield and bumper position and the roof angle are a percentage of the height or width.

$$
h_{w i}=K_{w i} h_{v} \quad h_{b}=K_{b} h_{v} \quad \alpha=K_{\alpha} w_{v}
$$

Then, the deformation parameter vector is:

$$
Z=\left\{y_{v}, K_{x}, K_{w}, K_{h}, K_{w i}, K_{b}, K_{\alpha}\right\}
$$




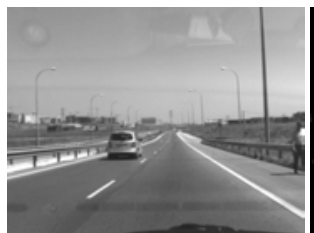

(a)

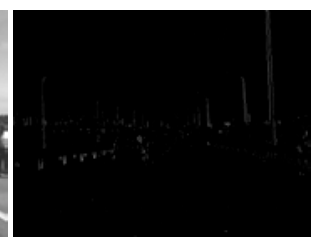

(b)

(b)

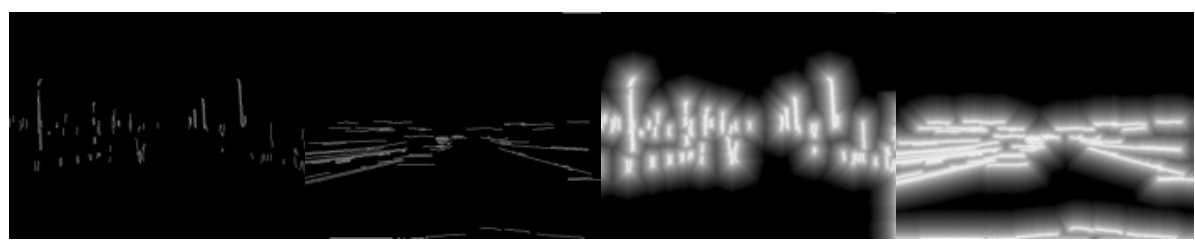

(c)

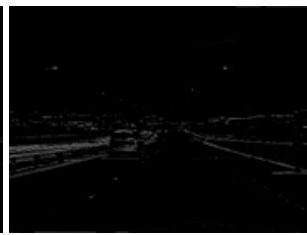

(d)

Fig. 2. Image processing (a) Image (b) Vertical and horizontal gradients (c) Vertical and horizontal edges $(\mathrm{d})$ vertical and horizontal distances

\subsection{Energy Function}

The en ergy function considers the following three factors: Symmetry, shape and the vehicle shadow (Fig. 2).

\section{Symmetry}

The symmetry of the vertical and horiz ontal edges is consi dered. For this reason, the vertical and hor izontal gradient components of the image are found (Fig. 2-b, Fig. 2c). Only the pixels with a high response in one of the components and low in the other are taken int o account. Then, the pairs of pi xels in the same line vote for the ce ntral pixel as their symmetry axe. The formulae can be found in [15].

\section{Shape}

Shape is defined by two energy terms: one based on the gradient (Fig. 2-b) and the other one base $\mathrm{d}$ on the distance to the edge $\mathrm{s}$, found before for the sy mmetry energy (Fig. 2-d). The formulae can be found in [15]. Here, only the distance formula is explained, because it has changed from the pr evious research. A distance image is obtained where each pixel shows the distance to the nearest ed ge. In order to emphasized the pixels that are near to the edges, the following look up table is applied

$$
\operatorname{Lut}(D)=\left\{\begin{array}{cc}
255\left(1-\operatorname{sqrt}\left(D / D_{\max }\right)\right) & 0<D<D_{\max } \\
0 & D>D_{\max }
\end{array} .\right.
$$

From that image, a distance to vertical edge en ergy, $D_{G V}$, and horizontal edge e nergy, $D_{G H}$, are calculated, where $D_{G}$ is the global distance energy.

$$
D_{G V}=\frac{1}{2 h}\left(\sum_{j=y}^{y+h} D v(x, j)+\sum_{j=y}^{y+h} D v(x+w, j)\right)
$$




$$
\begin{gathered}
D_{G H}=\frac{1}{4 w}\left(\sum_{i=x}^{x+w} D h(i, y)+\sum_{i=x}^{x+w} D h(i, y+t)+\sum_{i=x}^{x+w} D h(i, y+m)+\sum_{i=x}^{x+w} D h(i, y+h)\right) \\
D_{G}=\frac{\left(D_{G V}+D_{G H}\right)}{2} .(10)
\end{gathered}
$$

\section{Shadow}

The sh adow en ergy, $E_{S O M}$, of a vehicle wi th height $h$, width $w$, po sition $(x, y)$, and bumper position $m$, is defined by the average level of grey in the low er part of the model. Again, the formulae can be found in [15].

\section{Global Energy}

The final energy, $E$, is:

$$
E(Z)=-\left(k_{A} E_{\text {Sim }}(Z)+k_{B} E_{G}(Z)+k_{C} E_{D}(Z)+k_{D} E_{\text {Som }}(Z)\right)
$$

where $k_{A}, k_{B}, k_{C}$ and $k_{D}$ allow a weighted sum of the energy terms.

\subsection{Likelihood Probability Density Function}

The estimate of a give $\mathrm{n}$ deformation $Z$ for the image $I, P(I \mid Z)$, follows a Gibbs distribution [14]:

$$
P(I \mid Z)=\frac{1}{K} \exp -E(Z)
$$

where $K$ is the normalizing constant.

The detection problem is the search of the Maximum A Posteriori (MAP) estimation of $\mathrm{Z}$.

$$
Z_{M A P} \in \arg \max _{Z} P(I \mid Z) \in \arg \min _{Z} E(Z)
$$

The en ergy function is minimal wh en the deformed model ex actly matches with the one presented in the image.

\subsection{Search Algorithm}

Search algorithms have to find a balance between two opposite tasks: exploration of the complete search space and the exploitation of certain zones. With exploration, the search space is cove red looking for promising areas in which a more det ailed search has to be done; that is the exploitation task, where the best solution is looked for in a zone known as suitable. The risk is being trapped in a local maximum or minimum. Hashing methods a re the extreme case of exploration, where gradient-based methods (hillclimbing) are the extreme for exploitation.

Genetic algorithms (GAs) [16] do a parallel search in sev eral directions following an optimisation process, whi ch imitates natural selection and evolution. To accomplish this task, there is a set of possible solutions (the individuals) that ex change information depending on the fitness of the result in the search for the global maximum. GAs robustness relies in their ability to reach a global maximum surrounded by local ones. 


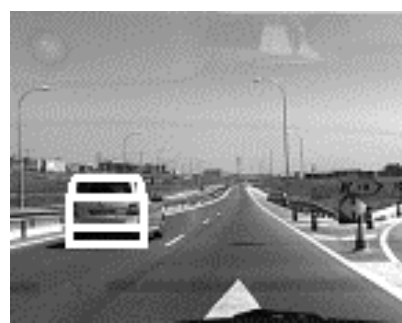

(a)

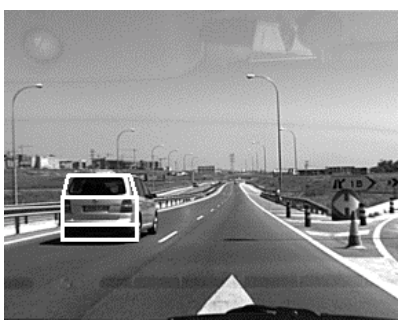

(b)

(c)

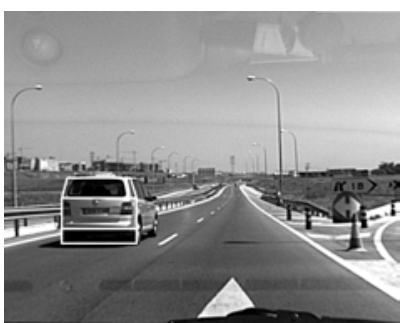

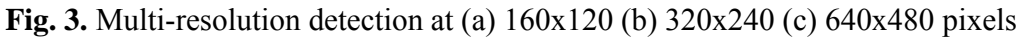

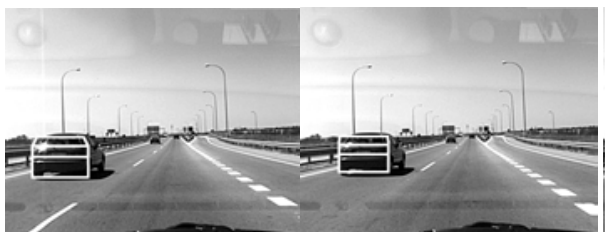

(a)

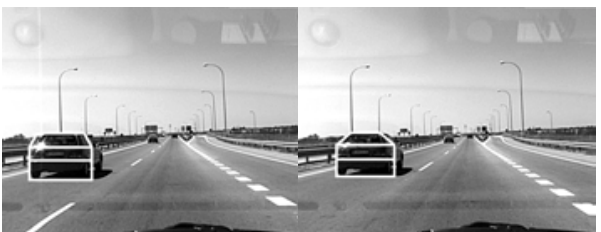

(b)

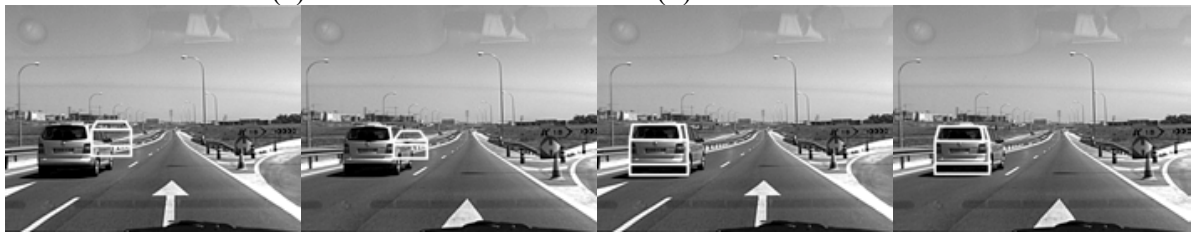

(c)

(d)

Fig. 4. Advantages of a mu lti-resolution approach. (a) small errors in the v ehicle detection (b) multi-resolution detection (c) wrong detection (d) multi-resolution detection

\section{Results}

The detection of the vehicle is d one for multiple resolutions. A Gaussian pyramid is built, with dimensions: 160x120,320x240, and 640x480 pixels. The information of the detection of lower levels is passed to greater levels (Fig.3). Working with a multiresolution approach has the main advantage of working with the best resolution for every circumstance. Take for example Fig. 4-a. The vehicle has been detected but, as there are many edges inside the car, there are some small errors in $\mathrm{t}$ he detection. Those edges inside the car have less importance at a lower resolution a nd the detection is b etter (Fig. 4-b). But, not on ly it is u seful to improve the results but also to detect successfully a vehicle. As the vehicle is in a cluttered environment, some edges in the environment can dece ive the alg orithm if an image $w$ ith g reat detail is used (Fig. 4-c). Again, working first with a smaller image improves the results (Fig. 4-d). Another advantage is the saving in computational time. In [15] 550 individuals were needed to detect the vehicles in front of the camera. With the present approach, only 32 individuals are needed. That means the algorithm spends now an average time for a genetic generation of $0.16 \mathrm{~ms}$ instead of the $24 \mathrm{~ms}$ of [15] (in a Pentium 4 Mobile at $1.7 \mathrm{GHz}$ ). The other parameters of the GA algorithm are: 


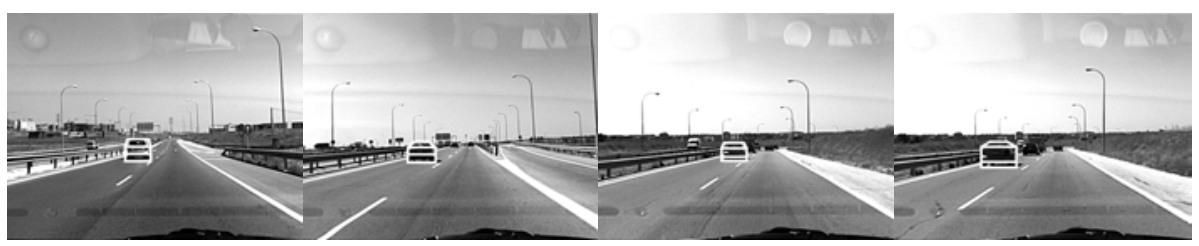

(a)

(b)

(c)

(d)

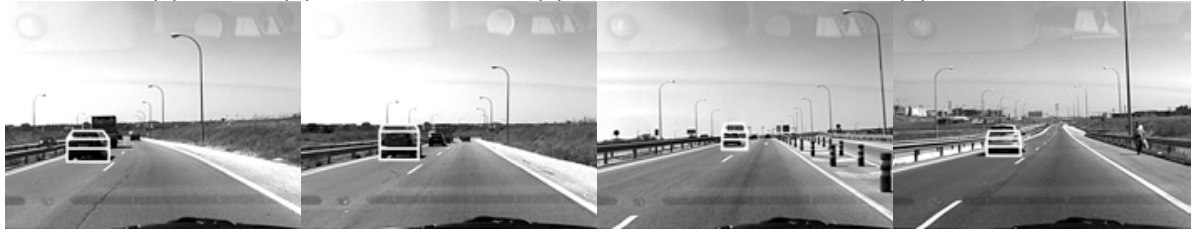

(e) (f)

(g)

(h)

Fig. 5. Some results and errors. (a)-(e) Successful detection of vehicles. (f)(g) Other rectangular objects are taken as part of the vehicle (h) an inner part of the vehicle is taken.

- Crossover probability: $70 \%$

- Mutation probability: 3\%

- Elitism

More results are shown, from Fig. 5-a to Fig. 5-e. Some errors are also shown. In Fig. 5-f-g, the vehicle detected is taller than the real one. This is because some rectangular objects in the environment, like buildings or informative signs are taking as part of the vehicle. Also, when the vehicle is very cl ose to the camera, a smaller vehicle is de tected (Fig. 5-h).

\section{Conclusions}

A system based on computer vision for the detection of other vehicles has been presented in this paper. It is based on a geometric model and its energy function includes information of the shape and symmetry of the vehicle and the sha dow it produces. A genetic algorithm has been used to find the optimum parameter values. The algorithm is able to detect vehicles in front of the camera, and it can also detect late ral vehicles and trucks.

\section{Acknowledgments}

This work was supported in part by the Spanish CICYT Grant TRA2004-07441-C0301 .

\section{References}

1. Dickmanns, E.D.: The development of machine vision for road vehic les in the last decade. IEEE Intelligent Vehicles Symposium (2002) 268-281

2. Charkari, N. M., Mori H.: Visual vehicle detection and tracking b ased on the sign pattern. Advanced Robotics 9 (1995) 367-382 
3. Hoffmann, C., Dang, T., Stiller, C.: Vehicle detection fusing 2D visual featur es. IEEE Intelligent Vehicles Symposium (2004) 280-285

4. Zielke, T., Brauckmann M., Von Seelen, W.: Intensity and edge-based symmetry detection with application to car-following. CVGIP: Image Understanding 58 (1993) 177-190

5. Goerick, C., Noll, D., Werner, M.: Artificial Neural Networks in Real Time Car detectio n and Tracking Applications. Pattern recognition Letters, 17, (1996) 335-343

6. Handmann, U., Kali nke, T., Tzo makas, C., Wer ner, M., Goerick C., von Seelen, W.: An image processin $\mathrm{g}$ system for driver assi stance. Image and Vision Computing 18 (2000) 367-376

7. ten Kat e, T .K., va n Leewen, M.B., Moro-E llenberger, S.E ., Dri essen, B.J. F., Ve rsluis, A.H.G., Groen, F.C.A.: Mid-range and Distant Vehicle Detection with a Mobi le Camera. IEEE Intelligent Vehicles Symposium (2004) 72-77

8. Matthews, N.D ., An, P.E., Roberts, J .M., Ha rris, C.J.: A neurof uzzy appro ach to future intelligent driver support system s. Proceedings-of the Institution of Mechanical Engineers Part D (Journal of Automobile Engineering) 212 (1998) 43-58

9. Broggi, A., Cerri, P., Antonello, P.C.: Multi-Resolution Vehicle Detection using Artificial Vision. IEEE Intelligent Vehicles Symposium (2004) 310-314

10. Sotelo, M.A., Fernandez, D., Naranjo, J.E., González, C., García, R., de Pedro, T., Reviejo, J.: Vision-based Adaptive Cruise Control for Intelligent Road Vehicles. IEEE/RSJ International Conference on Intelligent Robots and Systems (2004) 64-69

11. Betke, M., Harit aoglu, E., Davis, L. S.: Real-time mul tiple veh icle de tection and tracking from a moving vehicle. Machine Vision and Applications 12 (2000) 69-83

12. Ferryman, JM., Maybank S.J., Worrall, A. D.: Visual surveill ance for mov ing vehicl es. International Journal of Computer-Vision 37 (2000) 187-97

13. Kato, T., Ninomiya Y., Masak i I.: Precedi ng v ehicle re cognition based on lear ning from sample images, IEEE Transactions on Intelligent Transportation Systems 3 (2002) 252-260

14. Dubuisson, M-P., Lakshmanan S., Jain A.K.: Vehicle segmentation and classification using deformable templates. IEEE Transactions on Pattern analysis and Machine Int elligence 18 (1998) 293-308

15. Collado, J. M., Hilario, C., de 1 a Escaler a, A., Armingol, J. M M $^{\mathrm{a}}$.: Model Based Vehicle Detection for Intelligent Vehicles. IEEE Intelligent Vehicles Symposium (2004) 572-577

16. Goldberg, D.E.: Genetic Algorithms in Search, Optimization and Machine Learning. Addison-Wesley, Boston (1989) 